\title{
Plasticidad cerebral para el lenguaje en el niño y el adolescente
}

\author{
Juan Narbona, Nerea Crespo-Eguílaz
}

Introducción. La plasticidad posibilita el modelado estructural y funcional del sistema nervioso a lo largo de la vida por la experiencia, y fundamenta así los procesos de aprendizaje.

Objetivo. Revisar la literatura clínica y de resonancia magnética funcional (RMf) referente al remodelado de los sistemas cerebrales específicamente implicados en el lenguaje tras una lesión focal ocurrida desde el período perinatal hasta la adolescencia temprana.

Desarrollo. Cada uno de los grandes subsistemas neurocognitivos se desarrolla a diferentes ritmos; así, la plasticidad y la vulnerabilidad son diversas según la edad y el subsistema en los que ocurre una lesión cerebral focal. Al modificarse el programa genético local por lesiones, adquiridas durante el desarrollo, de áreas perisilvianas izquierdas, se ponen en marcha fenómenos de remodelado que desinhiben regiones homólogas contralaterales y activan, con mayor o menor fortuna, regiones vecinas homolaterales preservadas, según muestran los estudios con escucha dicótica, RMf y tractografía. Cuando la lesión focal ocurre en una edad prelingüística, o en la niñez temprana, el remodelado tisular permite un desarrollo lingüístico generalmente normal, a largo plazo. En las afasias por lesión adquirida a partir de los 5 años, la recuperación y el ulterior desarrollo del lenguaje son variables, con especial vulnerabilidad del acceso al léxico y de la lectoescritura.

Conclusión. El funcionalismo neurolingüístico posee subsistemas de reserva que permiten diferentes formas de plasticidad tras lesiones focales de las áreas específicas en el niño.

Palabras clave. Afasia infantil. Desarrollo lingüístico. Ictus. Neuroimagen funcional. Neuroplasticidad. Neurovulnerabilidad. Remodelado cerebral. Tractografía.

\section{Introducción}

El concepto de 'plasticidad neural' alude, en circunstancias normales, a la capacidad del sistema nervioso para modelar su estructura y su función con arreglo a la experiencia, lo que da lugar a los procesos de aprendizaje. Y, en circunstancias de pérdida patológica, a su capacidad para tratar de reactualizar las potencialidades del programa genético individual mediante fenómenos de remodelado. La dotación genómica permite, pues, un margen de adaptabilidad al manejar información y también al intentar compensaciones anatomofuncionales tras sufrir alguna agresión patógena [1,2].

La organización del sistema nervioso en la etapa extrauterina está regida por el código genético y por factores epigenéticos (factores de crecimiento, moléculas-guía, neurotransmisores...) en interacción con el medio externo: experiencia, educación, nutrición, respuesta a posibles lesiones... Los distintos dominios de las funciones cognitivas poseen una temporización de desarrollo morfofuncional propia para cada uno de ellos. Estudios con resonancia magnética funcional (RMf) han demostrado que en bebés de 2 meses de edad existe ya una activación predominante de la corteza temporal superior y frontal inferior del hemisferio izquierdo durante la escucha de material lingüístico, frente a la tarea base de escucha del mismo material acústico invertido [3]; aunque esta disposición es innata, el gradiente de dominancia perisilviana izquierda para el lenguaje aumenta y se consolida progresivamente hasta la niñez tardía [4].

Se pretende en este trabajo revisar los estudios clínicos neuropsicológicos y las investigaciones que, utilizando escucha dicótica, RMf o tractografía por imagen de tensor de difusión (DTI), permiten acercarse al conocimiento del remodelado de los sistemas cerebrales especialmente implicados en el lenguaje, tras una lesión focal, generalmente de naturaleza isquémica, ocurrida desde el período perinatal hasta la adolescencia temprana.

\section{Lenguaje tras lesiones unihemisféricas antes de los 2 años de edad}

Las lesiones congénitas limitadas a un hemisferio ce-
Unidad de Neuropediatría. Clínica Universidad de Navarra. Pamplona, Navarra, España.

Correspondencia:

Dr. Juan Narbona. Unidad de Neuropediatría. Departamento de Pediatría. Facultad de Medicina y Clínica Universidad de Navarra. Avda. Pío XII, s/n. E-31080 Pamplona (Navarra).

E-mail:

jnarbona@unav.es

Financiación:

Programa de ayuda a la investigación en Neurología del Desarrollo de la Fundación Fuentes Dutor/ICT, Pamplona.

Declaración de intereses: Los autores manifiestan la inexistencia de conflictos de interés en relación con este artículo.

Aceptado:

24.01.12.

Cómo citar este artículo: Narbona J, Crespo-Eguílaz N Plasticidad cerebral para el lenguaje en el niño y el adolescente. Rev Neurol 2012; 54 (Supl 1): S127-30.

(c) 2012 Revista de Neurología 
rebral, sea el izquierdo o el derecho, afectan a las capacidades cognitivas generales, tanto de índole verbal como no verbal, frente a controles; esto puede que no se haga evidente más que en estudios de seguimiento a largo plazo $[5,6]$. Pero la afectación cognitiva puede ser más global y tener más repercusión negativa sobre el rendimiento escolar y la conducta cuando la lesión recae sobre el hemisferio derecho [7]; probablemente las funciones genéticamente más ligadas a este hemisferio resultan más esenciales y menos sustituibles.

En otros trabajos -sobre casuísticas menos extensas, pero con mejor delimitación clínica y lesional, por resonancia magnética, de lesiones focales que afectan a los sistemas córtico-subcorticales del lenguaje en el hemisferio izquierdo, adquiridas desde el tercer trimestre intrauterino hasta los 2 años posnatales-, se ha comprobado una buena suplencia de las funciones lingüísticas por las zonas perilesionales respetadas en el hemisferio dañado y por sistemas homólogos contralaterales. En esta situación, aunque el desarrollo lingüístico primario se realiza a un ritmo retrasado, llega a ser normal a edad preescolar en casi la totalidad de los niños con parálisis cerebral hemiparética derecha [8-10].

Combinando los índices de dominancia hemisférica en tareas de escucha dicótica verbal con los datos de neuroimagen, en 26 escolares con una edad media de 7 años, afectos de hemiparesia congénita de uno $u$ otro hemicuerpos y con desarrollo intelectual y lingüístico normales, se encontró que sólo las lesiones córtico-subcorticales que implican al lóbulo temporal izquierdo se siguen de una dominancia hemisférica derecha en el test de escucha dicótica; en cambio, cuando la lesión afecta a otras áreas corticales del hemisferio izquierdo o se limita a la sustancia blanca periventricular, y el momento del daño cerebral fue antes del término gestacional, la lateralización funcional persiste en el hemisferio izquierdo $[8,9]$.

En 10 niños y adolescentes afectos de hemiplejía derecha connatal, con inteligencia normal y sin epilepsia resistente al tratamiento, de edades comprendidas entre 7 y 19 años (media: 10 años y 6 meses), se comprobó un índice de dominancia hemisférica derecha en la RMf durante una tarea de generación de rimas en ocho de los casos; los otros dos sujetos presentaban lesiones hemisféricas de pequeño tamaño alejadas de las estructuras córtico-subcorticales implicadas en los procesos de lenguaje [10]. Esta facilidad para cambiar la función lingüística al hemisferio derecho cuando la lesión hemisférica izquierda ocurre antes de finalizar el período perinatal no es tan evidente cuando la lesión es adquirida en edades posteriores, incluso en época preescolar.

\section{Remodelación del lenguaje por lesiones perisilvianas izquierdas adquiridas tras los 2 años de edad}

Existen varios estudios de caso único muy informativos y algunas pocas series multicéntricas de afasia adquirida por ictus infantojuvenil. El infarto cerebral en fase crónica, a pesar de su baja incidencia entre los 2 y los 16 años, es la lesión delimitada que mejor permite estudiar las correlaciones clinicopatológicas y los procesos de remodelación cerebral del lenguaje, frente a las entidades de otra naturaleza -traumática, neuroinfecciosa, tumoral, etc.- que, en cambio, conllevan daño cerebral la mayoría de las veces múltiple y difuso. En las series de ictus infantojuvenil la presencia de afasia se consigna con cierta frecuencia durante el período agudo hospitalario, pero su persistencia no es habitual en los escasos estudios de seguimiento a medio-largo plazo [11].

En trabajos clásicos se describían las afasias adquiridas durante la niñez con la característica común de hipofluidez y predominio de síntomas 'negativos' $[12,13]$. En contraste con la pobreza semiológica de estos primeros estudios, los trabajos de las últimas cuatro décadas han mostrado que, tanto en el período inmediato a la lesión como a lo largo del seguimiento, la afasia infantil puede presentarse bajo modalidades fluente o no fluente, con todos los síntomas 'negativos' (anartria, disprogramación fonológica, anomia, hipogramatismo, aprosodia...) y 'positivos' (jergafasia, parafasias semánticas...), que configuran el arco de variedades sindrómicas descritas en las afasias del adulto [14-20].

Los infartos perisilvianos izquierdos adquiridos entre los 3 y los 14 años tienen un curso de recuperación a corto-medio plazo mucho mejor que los adquiridos en adultos [11], pero pueden conservar sintomatología afásica leve a largo plazo. La comprensión y el discurso oral coloquiales son normales en la mayoría de casos, pero las observaciones longitudinales, varios años tras la lesión, denotan la presencia de dificultades importantes en el acceso al léxico y en las formas gramaticales complejas del discurso, así como en la comprensión y composición del lenguaje escrito. En una serie de 16 infartos isquémicos de estructuras subcorticales adquiridos en la niñez [19], los que afectaban a la región perisilviana izquierda cursaban con afasia no fluida durante las primeras semanas en todos los casos; la recuperación del lenguaje espontáneo y conversacional fue mejor cuando la lesión afectaba a estructuras anteriores (cabeza del caudado, pálido o brazo anterior de la cápsula interna) que cuando asentaba en el tálamo, el brazo posterior de la cápsula interna y el centro oval. 
Pavão-Martins et al [21] aportan la observación de una paciente de 6 años que, ocho meses después de un infarto córtico-subcortical frontal izquierdo con afasia de Broca, había recuperado aceptablemente la comprensión y la expresión excepto el acceso al léxico, que era disociado: la recuperación de verbos era normal, y la de nombres, significativamente baja respecto a controles. En estudios con RMf de participantes sanos con edades entre 7 y 32 años, se ha comprobado que la corteza frontal inferior izquierda se activa de forma creciente con arreglo a la edad en tareas de acceso al léxico (nombres y verbos), al tiempo que va decreciendo la activación proporcional de la corteza temporoparietal [21,22].

Cuando la lesión que origina la afasia se adquiere durante el período intermedio entre la niñez tardía y la adolescencia temprana (período peripuberal, de 10 a 16 años), la recuperación es mejor que en afasias del adulto, pero inferior a la observada en la casuística de niñez temprana y período escolar. Posee especial interés este intervalo etario, pues normalmente la adquisición del lenguaje es ya entonces completa, y, sin embargo, aún quedan bastantes procesos madurativos por realizarse en el cerebro.

Es destacable un estudio con RMf de siete pacientes con una edad media de 17 años, que habían sufrido afasia por infarto hemisférico izquierdo con una edad media de 10 años [23]. Durante las tareas de comprensión de morfemas gramaticales y de generación subvocal de rimas, el mapeo por RMf mostró una activación de ambos hemisferios; el índice de dominancia de activación era hacia el hemisferio izquierdo en los pacientes que poseían un mejor nivel de lenguaje. No obstante, el estudio de los mismos pacientes tres años después [24], sin haber recibido tratamiento rehabilitador específico durante este período, mostró que todos habían hecho un progreso suplementario en sus competencias lingüísticas y que éste se correlacionaba positivamente con el grado de activación del hemisferio derecho en la RMf. Estos dos estudios sobre sujetos con afasia adquirida tras el pleno desarrollo del leguaje, pero sin estar concluida la maduración cerebral, abren el debate sobre el papel de cada uno de los hemisferios en el remodelado del leguaje en edad adolescente. Es probable que la desinhibición de las estructuras del hemisferio derecho homólogas a las normalmente programadas en el hemisferio izquierdo para el lenguaje desempeñe un importante papel en la recuperación clínica durante mucho más allá del primer año tras la lesión. Pero también hay que considerar, con arreglo a los trabajos con RMf repetida a lo largo de años en pacientes juveniles o adultos, que la recuperación ulterior del lenguaje acaba reclutando estructuras del hemisferio iz- quierdo periféricas a la lesión [25-28]. La tractografía por DTI ha permitido poner de manifiesto que el fascículo arcuato madura normalmente sus conexiones y su mielinización desde la niñez hasta la edad adulta [26,27]; tras una lesión perinatal bilateral de la porción superior del fascículo arcuato, la tractografía por DTI ha permitido observar un mayor desarrollo de la porción inferior directa frontotemporal de dicho fascículo a través de la cápsula extrema, en un escolar con desarrollo lingüístico normal [28].

\section{A modo de síntesis}

Cada uno de los grandes subsistemas neurocognitivos se desarrolla a diferentes ritmos; así, la plasticidad y la vulnerabilidad son diversas según la edad y el subsistema en los que ocurre una lesión cerebral focal; por ejemplo, las estructuras parietooccipitales que soportan funciones de integración visuoespacial maduran antes, y son más vulnerables a edades tempranas, que la región prefrontal y las funciones ejecutivas, con un curso de desarrollo más tardío y prolongado. El funcionalismo neurolingüístico posee subsistemas de reserva que permiten diferentes grados de recuperación y desarrollo tras lesiones focales de las áreas específicas del hemisferio cerebral izquierdo en el niño. Tras modificarse el programa genético local por una lesión de las áreas perisilvianas izquierdas, adquirida durante el desarrollo, se ponen en marcha fenómenos de remodelado que desinhiben regiones homólogas contralaterales y activan, con mayor o menor fortuna, regiones vecinas homolaterales preservadas, según muestran los estudios con escucha dicótica, con RMf y con tractografía. La mayor parte de los trabajos permite estimar que, si la lesión focal ocurre en edad prelingüística, o incluso en la niñez temprana, el remodelado tisular posibilita un desarrollo lingüístico generalmente normal, a largo plazo. En las afasias por lesión adquirida a partir de los 5 años la recuperación y el ulterior desarrollo del lenguaje son variables, con especial vulnerabilidad del acceso al léxico y de la lectoescritura. La RMf con tareas lingüísticas y la tractografía por DTI están permitiendo avanzar en el conocimiento de la plasticidad neural, alterada por lesiones focales de las áreas del lenguaje durante el desarrollo madurativo, al relacionar las capacidades lingüísticas recuperadas con el grado y tipo de remodelaciones cerebrales.

\section{Bibliografía}

1. Anderson V, Spencer-Smith M, Wood A. Do children really recover better? Neurobehavioural plasticity after early brain insult. Brain 2011; 134: 2197-221. 
2. Rimrod SL, Johnston MV. Neuronal plasticity and developmenta disabilities. In Shevell M, ed. Neurodevelopmental disabilities: clinical and scientific foundations. International Review of Child Neurology Series. London: Mac Keith Press; 2009. p. 225-40.

3. Deahaene-Lambertz G, Dehaene S, Hertz-Panier L. Functional neuroimaging of speech perception in infants. Science 2002; 298: 2013-5.

4. Ahmad Z, Balsamo LM, Sachs BC, Xu B, Gaillard WD. Auditory comprehension of language in young children. Neural networks identified with fMRI. Neurology 2003; 60: 1598-605.

5. Gabari-Gambarte I. Desarrollo neuropsicológico en sujetos con lesión cerebral unihemisférica congénita. Rev Psicol Gral Aplic 1995; 48: 301-14.

6. Westmacott R, MacGregor D, Askalan R, DeVeber G. Late emergence of cognitive deficits after unilateral neonatal stroke. Stroke 2009; 40: 2012-9.

7. Westmacott R, Askalan R, MacGregor D, Anderson P, DeVeber G. Cognitive outcome following unilateral arterial ischaemic stroke in childhood: effects of age and lesion location. Dev Med Child Neurol 2010; 52: 386-93.

8. Chilosi AM, Pecini C, Cipriani P, Brovedani P, Brizzolara D, Ferretti G, et al. Atypical language lateralization and early linguistic development in children with focal brain lesions. Dev Med Child Neurol 2005; 47: 725-30.

9. Brizzolara D, Pecini C, Brovedani P, Ferretti G, Cipriani P, Cioni $\mathrm{G}$. Timing and type of congenital brain lesion determine different patterns of language lateralization in hemiplegic children. Neuropsychologia 2002; 40: 620-32.

10. Bulgheroni S, Nichelli F, Erbetta A, Bagnasco I, Riva D. Verbal dichotic listening and manual performance in children with congenital unilateral brain lesions. Neuropsychologia 2004; 18: 748-55.

11. Guzzetta A, Pecini C, Biagi L, Tosetti M, Brizzolara D, Chilosi A, et al. Language organization in left perinatal stroke. Neuropediatrics 2008; 39: 157-63.

12. Narbona J, Crespo-Eguílaz N. Afasia en niños y adolescentes: aspectos evolutivos. Rev Neurol 2008; 46 (Supl 1): S87-9.

13. Alajouanine T, Lhermitte F. Acquired aphasia in children. Brain 1965; 88: 653-62.

14. Woods MT, Teuber HL. Changing patterns of childhood aphasia. Ann Neurol 1978; 3: 273-80.

15. Van Hout A, Lyon G. Wernicke aphasia in a ten year old boy. Brain Lang 1986; 29: 268-85.

16. Martins JP, Ferro JM. Acquired conduction aphasia in a child Dev Med Child Neurol 1987; 29: 532-6.
17. Hynd GW, Leathem J, Semrud-Clikeman M, Hern KL, Wenner M. Anomic aphasia in childhood. J Child Neurol 1995; 10: 289-93.

18. Van Hout A, Evrard P, Lyon G. On the positive semiology of acquired aphasia in children. Dev Med Child Neurol 1985; 27: 231-41.

19. Van Dongen HR, Paquier PF, Creten WL, Van Borsel J, Catsman-Berrevoets CE. Clinical evaluation of conversational speech fluency in the acute phase of acquired childhood aphasia: does a fluency/nonfluency dichothomy exist? J Child Neurol 2001; 16: 345-51.

20. Gout A, Seibel N, Rouvière C, Husson B, Hermans B, Laporte N, et al. Aphasia owing to subcortical brain infarcts in childhood. J Child Neurol 2005; 20: 1003-8.

21. Pavão-Martins I, Loureiro C, Ramos S, Moreno T. Grammatical dissociation during acquired childhood aphasia. Dev Med Child Neurol 2009, 51: 999-1002.

22. Shapiro KA, Moo LR, Caramazza A. Cortical signatures of noun and verb production. Proc Natl Acad Sci U S A 2006; 103: 1644-9.

23. Brown TT, Lugar HM, Coalson RS, Miezin FM, Petersen SE Schlaggar BL. Developmental changes in human cerebral functional organization for word generation. Cereb Cortex 2005; 15: 275-90.

24. Elkana O, Frost R, Kramer U, Ben-Bashat D, Hendler T, Schmidt D, et al. Cerebral reorganization as a function of linguistic recovery in children: an fMR study. Cortex 2011: 47: 202-16.

25. Elkana O, Frost R, Kramer U, Ben-Bashat D, Schweiger A. Cerebral language reorganization in the chronic stage of recovery: a longitudinal fMRI study. Cortex 2011. [Epub ahead of print].

26. Anderson V, Jacobs R, Spencer-Smith M, Coleman L, Anderson $\mathrm{P}$, Williams J, et al. Does early age at brain insult predict worse outcome? Neuropsychological implications. J Pediatr Psychol 2010; 35: 716-27.

27. Catani M, Thiebaut de Schotten M. A diffusion tensor imaging tractography atlas for virtual in vivo dissections. Cortex 2008; 44: 1105-32.

28. Brauer J, Anwander A, Friederici AD. Neuroanatomical prerequisites for language functions in the maturing brain Cereb Cortex 2011; 21: 459-66.

28. Yeatman JD, Feldman HM. Neural plasticity after pre-linguistic injury to the arcuate and superior longitudinal fasciculi. Cortex 2011. [Epub ahead of print].

\section{Brain plasticity for language in children and adolescents}

Introduction. Plasticity makes possible adaptative modelling of the nervous system to experiences i.e. learning and development.

Aim. To review current literature on clinical long term evolution and functional magnetic resonance imaging (fMRI) features of brain remodelling after focal stroke in left perisylvian regions involved in basic language processing during infancy and childhood.

Development. Each of the main neurocognitive subsystems develops with different timing, so altered plasticity and vulnerability are diverse, according with age at insult and its topography. Genetic programming makes human brain capable for installing basic formal linguistic abilities on an associative perisylvian subsystem, highly specialised. A focal lesion of this region leads to remodelling phenomena by disinhibition of contralateral frontal and perisylvian structures and by a more or less efficacious activation of neighboring homolateral cortex, as it has been shown by fMRI studies and DTI tractography. As a result, very early local stroke to language areas is generally well compensated in terms of linguistic behaviour. Meanwhile acquired aphasias into middle and late childhood, even if they have a better prognosis than in adults, they fail to resume without lexical access defaults and/or difficulties in written language.

Conclusion. Brain plasticity can promote restoration and further development of language following a stroke in left perisylvian areas, specially when lesion occurs at perinatal to middle childhood.

Key words. Brain remodelling. Child aphasia. Functional neuroimaging. Language development. Neuroplasticity. Neurovulnerability. Stroke. Tractography. 